\title{
On the Possible Temperatures of a Dynamical System
}

\author{
Ola Bratteli ${ }^{1}$, George A. Elliott ${ }^{2}$, and Richard H. Herman ${ }^{3 \star}$ \\ ${ }^{1}$ Mathematics Institute, University of Oslo, Norway and Centre de Physique Théorique II, CNRS, \\ Marseille, France \\ ${ }^{2}$ Mathematics Institute, University of Copenhagen, Denmark, and Department of Mathematics, \\ University of Ottawa, Canada \\ ${ }^{3}$ Department of Mathematics, Pennsylvania State University, Pennsylvania, PA 16802, USA
}

\begin{abstract}
A simple $C^{*}$-algebra and a continuous one-parameter automorphism group are constructed such that the set of inverse temperatures at which there exist equilibrium states (i.e., KMS states, or, for $\beta= \pm \infty$, ground or ceiling states) is an arbitrary closed subset of $\mathbb{R} \cup\{ \pm \infty\}$.
\end{abstract}

\section{Introduction}

It is well known that the Fermion $C^{*}$-algebra with the gauge automorphism group has KMS states at all inverse temperatures, and also that for a general $C^{*}$-algebra with unit the set of inverse temperatures at which KMS states for a given oneparameter automorphism group occur is a closed subset of $\mathbb{R} \cup\{ \pm \infty\}$ (see $[2,17]$.

The $C^{*}$-algebra $O_{n}$ studied by Cuntz in [4] has a one-parameter automorphism group of period $2 \pi$ with a KMS state at the unique inverse temperature $\log n, n=1,2, \ldots \infty$. (For a proof of this see [2] or [11].)

Here we construct a simple $C^{*}$-algebra and a one-parameter automorphism group of period $2 \pi$ with KMS states at inverse temperatures in any given closed subset of $\mathbb{R} \cup\{ \pm \infty\}$, and only at these inverse temperatures. The construction used is related to both of the above, and in fact coincides with the first one in that case. This suggests the question as to whether or not it has a physical interpretation.

This construction settles a question of Sakai [17], as to whether or not the set of temperatures need be a convex subset of $\mathbb{R}$.

\section{An Automorphism Scaling Traces}

2.1. Theorem. Let $F$ be a closed subset of the unit interval $[0,1]$, and denote by $\mathbb{Z}[x]_{F}$ the group $\mathbb{Z}[x]$ of polynomials over $\mathbb{Z}$ ordered by the following relation:

$p>0$ if $p(t)>0$ for all $0<t<1$ in a neighbourhood of $F$.

Then $\mathbb{Z}[x]_{F}$ is a dimension group.

* With partial support of the National Science Foundation 
Proof. First note that $\mathbb{Z}[x]_{F}$ obviously has the property that $n a>0$ for some $n=2,3, \ldots$ implies $a>0$. Hence, by [5], it is sufficient to verify that $\mathbb{Z}[x]_{F}$ has the Riesz interpolation property. Recall that an ordered group has the Riesz interpolation property if $a, b \geqq c, d$ implies that for some $e, a, b \geqq e \geqq c, d$ (see [8]).

To show that $\mathbb{Z}[x]_{F}$ has the Riesz interpolation property we shall reduce the problem to the case that $F$ is the whole unit interval $[0,1]$, in which case the problem has been solved by Renault in [14, Appendix]. We shall show that if $a, b \geqq c, d$ in $\mathbb{Z}[x]_{F}$, then there exist $a^{\prime} \leqq a$ and $b^{\prime} \leqq b$ in $\mathbb{Z}[x]_{F}$ such that, in $\mathbb{Z}[x]_{[0,1]}$ (recall that the underlying group is the same), $a^{\prime}, b^{\prime} \geqq c, d$. Then, of course, if $a^{\prime}, b^{\prime} \geqq e$ $\geqq c, d$ in $\mathbb{Z}[x]_{[0,1]}$, this inequality holds also in $\mathbb{Z}[x]_{F}$.

By the theorem of Weierstrass, any continuous function on $[0,1]$ can be approximated uniformly by polynomials. Moreover, if the function has integral values at both 0 and 1 , the approximating polynomials may be chosen to have integral coefficients ${ }^{1}$. To see this we proceed as follows. Since $f$ has integer values at 0 and 1 we can assume $f(0)=f(1)=0$. As we wish only to approximate $f$ we can further assume $f$ is zero in a neighbourhood of 0 and 1. We then apply the ordinary Weierstrass theorem to $\frac{f(x)}{[x(1-x)]^{n}}$, where $n$ is to be chosen later. We obtain a polynomial, $\sum_{i=0}^{l} a_{i} x^{i}$ such that

$$
\left|\frac{f(x)}{[x(1-x)]^{n}}-\sum_{i=0}^{l} a_{i} x^{i}\right| \leqq \frac{1}{2} \text { for all } x \in[0,1] .
$$

We can then rewrite this polynomial as $\sum_{i=0}^{l}\left(b_{i}+c_{i} x\right)(x(1-x))^{i}$, so that

$$
\left|\frac{f(x)}{[x(1-x)]^{n}}-\sum_{i=0}^{l}\left(b_{i}+c_{i} x\right)(x(1-x))^{i}\right| \leqq \frac{1}{2} \quad \text { on }[0,1] \text {. }
$$

Replacing $b_{i}$ and $c_{i}$ by their integral parts, $\left[b_{i}\right]$ and $\left[c_{i}\right]$, we obtain a polynomial which differs from the original by at most $\sum_{0}^{l} 2 \cdot\left(\frac{1}{4}\right)^{i}$, so that

$$
\left|\frac{f(x)}{[x(1-x)]^{n}}-\sum_{i=0}^{l}\left(\left[b_{i}\right]+\left[c_{i}\right] x\right)(x(1-x))^{i}\right| \leqq 4 .
$$

Multiplying through by $[x(1-x)]^{n}$ we obtain

$$
\left|f(x)-x^{n}(1-x)^{n} \sum_{i=0}^{l} d_{i} x^{i}\right| \leqq\left(\frac{1}{4}\right)^{n} 4 \text { on }[0,1]
$$

with the $d_{i}$ integers. Now as $n$ was arbitrary we are done.

Consequently, for any $\varepsilon>0$ and $m \in \mathbb{R}$ there exists $p \in \mathbb{Z}[x]$ such that:

$p(t)<\varepsilon$ for all $t \in[0,1]$;

$p(t)<m$ for all $t$ in a specified compact subset of $[0,1] \backslash F$;

$p(t)<0$ for all $0<t<1$ in a neighbourhood of $F$.

1 Results of this type are well known. For a survey see a forthcoming A.M.S. memoir by LeBaron O. Ferguson 
To see this, choose a continuous function $f$ on $[0,1]$ such that for some $r, s=0,1,2, \ldots$ :

$$
\begin{aligned}
& f(t)=1 \text { for } t=0 \text { if } 0 \in F, \text { and for } t=1 \text { if } 1 \in F ; \\
& f(t) t^{r}(1-t)^{s}<2 \varepsilon / 3 \text { for all } t \in[0,1] ; \\
& f(t) t^{r}(1-t)^{s}<m-\varepsilon / 3 \text { for all } t \text { in the specified compact subset of }[0,1] \backslash F ; \\
& f(t)>\varepsilon / 3 \text { for all } 0<t<1 \text { in a neighbourhood of } F .
\end{aligned}
$$

Approximate $f$ within $\varepsilon / 3$ on $[0,1]$ by $p^{\prime} \in \mathbb{Z}[x]$; then $p^{\prime} x^{r}(1-x)^{s}$ satisfies the requirements for $p$.

It follows that if $a_{i}>b_{j}$ in $\mathbb{Z}[x]_{F}, i, j=1,2$, there exists $q \in \mathbb{Z}[x]$ such that $q>0$ in $\mathbb{Z}[x]_{F}$ and $a_{i}-q>b_{j}$ in $\mathbb{Z}[x]_{[0,1]}, i, j=1,2$. To see this, consider the continuous function $g$ defined on $[0,1]$ by

$$
g(t)=\max _{i, j}\left(a_{i}-b_{j}\right)(t) / t^{r}(1-t)^{s},
$$

where $r$ and $s(=0,1,2, \ldots)$ are chosen so that $g(0)$ and $g(1)$ are finite and not zero. Then for some $\varepsilon>0$ and some neighbourhood $N$ of $F$ in $[0,1]$,

$$
g(t)>\varepsilon \text { for all } t \in N .
$$

By the previous paragraph, choose $p \in \mathbb{Z}[x]$ such that, with $m=\inf _{t \in[0,1]} g(t)$ :

$$
\begin{array}{ll}
p(t)<\varepsilon \quad \text { for all } & t \in[0,1] ; \\
p(t)<m & \text { for all } t \in[0,1] \backslash N ; \\
p(t)>0 & \text { for all } \quad 0<t<1 \quad \text { in a neighbourhood of } F .
\end{array}
$$

Then $p(t)<g(t)$ for all $t \in[0,1]$, and hence $p x^{r}(1-x)^{s}$ satisfies the requirements for $q$.

Hence by $[14$, Appendix $]$ there exists $e \in \mathbb{Z}[x]$ with $a_{i}-p \geqq e \geqq b_{j}$ in $\mathbb{Z}[x]_{[0,1]}$, and then $a_{i} \geqq e \geqq b_{j}$ in $\mathbb{Z}[x]_{F}$. This shows that $\mathbb{Z}[x]_{F}$ has the Riesz interpolation property.

2.2. Corollary. Let $F$ be a closed subset of the unit interval $[0,1]$. Denote by $G$ the group $\mathbb{Z}\left[x, x^{-1},(1-x)^{-1}\right]$ of polynomials in $x, x^{-1}$ and $(1-x)^{-1}$ over $\mathbb{Z}$, and by $G_{F}$ the ordered group defined by the order relation in $G$ :

$$
p>0 \text { if } p(t)>0 \text { for all } 0<t<1 \text { in a neighbourhood of } F .
$$

Then $G_{F}$ is a dimension group.

Proof. $G_{F}$ is the inductive limit of the sequence

$$
\mathbb{Z}[x]_{F} \rightarrow \mathbb{Z}[x]_{F} \rightarrow \ldots,
$$

where each map consists of multiplication by $x(1-x)$.

It can be shown, from Shen's local criterion [16], and also follows easily from the characterization given in [5], that the class of dimension groups is closed under inductive limits. 
2.3. Problem. Renault showed in [14] that Pascal's triangle is a Bratteli diagram for $\mathbb{Z}[x]_{[0,1]}$. This shows that this dimension group has a diagram with injective embedding matrices. Is this also true for $\mathbb{Z}[x]_{F}$ ?

2.4. Theorem. Let $F$ be a closed subset of the unit interval $[0,1]$, and consider the dimension group $G_{F}$ of 2.2. Consider the automorphism $\alpha$ of the underlying group $G$ consisting of multiplication by $x(1-x)^{-1}$. Then $\alpha$ is an order automorphism of $G_{F}$, and for each $t \in F$, if $e_{t}: G_{F}^{+} \rightarrow \mathbb{R}^{+} \cup\{+\infty\}$ denotes the positive-additive map $a \mapsto a(t)$, then

$$
e_{t} \alpha=\frac{t}{1-t} e_{t}
$$

Conversely, if $\varphi: G_{F}^{+} \rightarrow \mathbb{R}^{+} \cup\{+\infty\}$ is a positive, additive map such that $\varphi(1)=1$ and for some $s \in \mathbb{R}^{+} \cup\{+\infty\}, \varphi \alpha=s \varphi$, then, with $t=s(1+s)^{-1}$, $t$ belongs to $F$ and $\varphi=e_{t}$.

Proof. Let $\varphi: G_{F}^{+} \rightarrow \mathbb{R}^{+} \cup\{+\infty\}$ be a positive, additive map with $\varphi(1)=1$, such that $\varphi \alpha=s \varphi$ (where $+\infty \cdot 0$ is indeterminate), and consider first the restriction of $\varphi$ to $\mathbb{Z}[x]_{F}^{+} \subset G_{F}^{+}$; denote this restriction by $\psi$. Then $\psi$ is finite valued, positive and $\psi(1)=1$; hence $\psi$ extends to a functional on $\mathbb{Z}[x]_{F}$ continuous with respect to the supremum norm. By the proof of 2.1 , the closure of $\mathbb{Z}[x]_{F}$ in this norm consists of the continuous functions on $F$ with integral values at 0,1 (if either of these points belongs to $F$ ). This shows that $\psi$ is determined by a measure on $F$; it is clear that this measure must be concentrated at a point.

We must now show that $\varphi$ is uniquely determined on all of $G_{F}^{+}$; we shall use that its restriction to $\mathbb{Z}[x]_{F}^{+}$is determined. We note that any element of $G_{F}^{+}$may be written as $p x^{-r}(1-x)^{-r}$ where $p \in \mathbb{Z}[x]_{F}^{+}$and $r=0,1,2, \ldots$ (cf. 2.2). In view of the expansion

$$
x^{-r}(1-x)^{-r}=\left(x^{-1}+(1-x)^{-1}\right)^{r}=x^{-r}(1-x)^{-r}+\sum_{k=1}^{r-1} x^{-k}(1-x)^{-r+k}
$$

we see by induction on $r$ that, in order to show that $\varphi$ is determined on all of $G_{F}^{+}$, it is enough to show that it is determined on elements of the form $q x^{-k}$ or $q(1-x)^{-k}$ where $q \in \mathbb{Z}[x]_{F}^{+}$and $k=1,2, \ldots$. Since

$$
\begin{aligned}
q x^{-k} & =q x^{-k+1}+\alpha^{-1}\left(q x^{-k+1}\right), \\
q(1-x)^{-k} & =q(1-x)^{-k+1}+\alpha\left(q(1-x)^{-k+1}\right),
\end{aligned}
$$

and $\varphi \alpha^{ \pm 1}=s^{ \pm 1} \varphi$, this last holds by induction on $k$.

\section{KMS States for the Dual Automorphism Group}

3.1. Let $F$ be a non-empty closed subset of the unit interval $[0,1]$, and denote by $A=A_{F}$ the separable approximatively finite-dimensional $C^{*}$-algebra, unique up to isomorphism (see [6]), whose dimension range is the positive part of the dimension group $G_{F}$ of 2.2. By [6] the automorphism $\alpha$ of $G_{F}$ defined in 2.2 is induced by an automorphism of $A$; we shall choose one and denote it also by $\alpha$.

Furthermore, we will choose $\alpha$ in the following way. Let $e \in A_{F}$ be a fixed projection in the equivalence class corresponding to $1 \in G_{F}$. Since $x<1$, in the 
ordering on $G_{F}$, there exists a projection $g \in A_{F}$, corresponding to $x$, such that $g \leqq e$ (in the usual ordering of self-adjoint operators). One has $\alpha^{-1}(x)=1-x \leqq 1$ in the ordering on $G_{F}$, and hence $\alpha^{-1}(g) \preccurlyeq e$ in the Murray-von Neumann ordering, whatever the choice of $\alpha$ is. Thus by modifying $\alpha^{-1}$ by an automorphism implemented by a unitary operator in the multiplier algebra of $A_{F}$, we may assume $\alpha^{-1}(g) \leqq e$ in the usual ordering of self-adjoint operators. In what follows we will assume $\alpha$ has been so modified.

We need some comments on the ideal structure of $A$. Ideals in $G_{F}$ are in one to one correspondence with ideals of $A$. If $F$ does not contain 0 or 1 then there are no ideals in $G_{F}$ and so $A$ is simple. If either or both of 0 or 1 are in $F$ then the only ideals are those generated by a projection corresponding to an integral power of $x$ or $1-x$, or a product of such powers. This may be seen as follows. One can map $G_{[0,1]}$ onto $G_{F}$ via the (positive) identity map for the underlying group. An ideal of $G_{F}$ then comes from an ideal in $G_{[0,1]}$ and these ideals are exactly what we claim they are, since the diagram for $G_{[0,1]}$ is

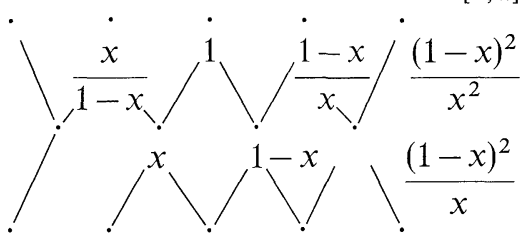

This is seen by combining the result of Renault [ibid] and Bratteli [1].

Note that no ideal is invariant under the automorphism $\alpha$ and its inverse.

Alternately we can see that the possible ideals in $A_{F}$ are of the form $I_{n, m}$ where $n, m$ run through $\mathbb{Z} \cup\{+\infty\}$. If $p \in G_{F}$, then $p \in I_{n, m}$ (remember that ideals in $G_{F}$ correspond to ideals in $A_{F}$ ) if and only if the following conditions are fulfilled.

a) If $1 \in F$ and $n<+\infty$ then $|p(x)|=0\left(|1-x|^{-n}\right)$ as $x \rightarrow 1$.

b) If $0 \in F$ and $m<+\infty$ then $|p(x)|=0\left(|x|^{-m}\right)$ as $x \rightarrow 0$.

All ideals except $\{0\}$ are of this form, in particular $I_{\infty, \infty}=A_{F}$. If $1 \notin F$ then $I_{n, m}\left(=I_{\infty, m}\right)$ is independent of $n$. A similar remark holds if $0 \notin F$. Since $I_{n, m}=I_{\infty, m} \cap I_{n, \infty}$, the prime ideals are $\{0\}, I_{n, \infty}, I_{\infty, m}$ where $n, m \in \mathbb{Z} \cup\{+\infty\}$. Again we see that $A_{F}$ is simple if neither 0 or $1 \in F$. Note that $\alpha$ maps $I_{n, m}$ onto $I_{n+1, m-1}$.

In general there does not exist a hereditary sub- $C^{*}$-algebra of $A$ invariant under $\alpha$ such that the restrictions to this subalgebra of the extreme traces of $A$ are finite. (Such a subalgebra does exist if $F$ lies entirely in the subinterval $\left[0, \frac{1}{2}[\right.$, but even then it cannot be chosen to be unital or to be invariant under $\alpha^{-1}$ too.) Thus, the dual weights of the traces which are scaled by $\alpha$, although they are KMS weights for the dual automorphism group, will not be finite.

Of course one could proceed using the well developed theory of KMS weights (see $[3,19]$ ), but instead of proceeding in this way, partly since there are technical difficulties in proving uniqueness of KMS weights on the whole crossed product of $A_{F}$ by $\alpha$, and especially because for infinite inverse temperatures they are in fact not unique (see 3.3), we shall cut down the crossed product by the projection $e \in A_{F}$ in the (convenient) equivalence class corresponding to $1 \in G_{F}$.

The advantage of considering the $C^{*}$-algebra $B=B_{F}$ obtained by cutting down the crossed product by a projection in this particular equivalence class is that one obtains uniqueness of KMS states even for infinite inverse temperatures (cf. 3.2). 
We have already noted that there are no $\alpha$-invariant ideals in $A$, and, as $\alpha$ transforms each non-zero projection in $A$ into an inequivalent projection, $\alpha$ is properly outer in the sense of [7]. The $C^{*}$-crossed product $C^{*}(A, \alpha)$ is simple by 3.2 of [7] as it coincides with the reduced crossed product considered there since the integers are amenable. The hereditary subalgebra $B$ of $C^{*}(A, \alpha)$ is then simple. The approximately finite-dimensional $C^{*}$-algebra $B \cap A$ has dimension range the order interval $[0,1]$ in the dimension group $G_{F}$ of 2.2. If $u$ denotes a fixed unitary multiplier of the crossed product of $A$ by $\alpha$ determining the automorphism $\alpha$ of $A$, then $B$ is the closed linear span of those elements $a u^{n}, a \in A, n \in \mathbb{Z}$, with support and range contained in the unit of $B$. We shall denote the restriction to $B$ of the dual automorphism group by $\gamma$, so that for each $z \in \mathbb{T}, \gamma_{z}\left(a u^{n}\right)=z^{n} a u^{n}$.

3.2. Theorem. Let $-\infty \leqq \beta \leqq+\infty$. Then there exists a KMS state on $B$ for the automorphism group $\varrho: \mathbb{R} \ni t \rightarrow \gamma_{\text {expit }}$ at the inverse temperature $\beta$ if and only if $e^{-\beta}\left(1+e^{-\beta}\right)^{-1} \in F$. In this case such a state is unique.

Proof. By 2.4 there exists a trace $\varphi$ on $A$ such that $\varphi \alpha=e^{-\beta} \varphi$ and $\varphi(e)=1$ if and only if $e^{-\beta}\left(1+e^{-\beta}\right)^{-1} \in F$. We pick such a $\beta$, and normalize the corresponding $\varphi$ such that $\varphi(e)=1$, where $e \in A$ is the unit for $B$. Let $\varepsilon$ denote the projection from $B$ onto $A \cap B$ given by $\varepsilon(x)=\int_{\mathbb{T}} \gamma_{z}(x) d z$ and define a state $\psi$ on $B$ by $\psi=\varphi \varepsilon$. We shall show that $\psi$ is a KMS state for the automorphism group $\varrho_{t}=\gamma_{\exp (i t)}$ of $B$ at the inverse temperature $\beta$. Assume first that $\beta$ is finite. It is enough by linearity and continuity to show that for each $x, y \in\left\{a u^{n} \mid a \in A, a u^{n} \in B, n \in \mathbb{Z}\right\}$, one has that

$$
\psi\left(y \varrho_{i \beta}(x)\right)=\psi(x y),
$$

[2]. If $x=a u^{n}, y=b u^{m}$, one has $y \varrho_{i \beta}(x)=b u^{m} e^{-n \beta} a u^{n}=e^{-n \beta} b \alpha^{m}(a) u^{m+n}$ and $x y$ $=a \alpha^{n}(b) u^{n+m}$. Thus $\psi\left(y \varrho_{i \beta}(x)\right)=\psi(x y)=0$ unless $m=-n$, and then

$$
\begin{aligned}
\psi\left(y \varrho_{i \beta}(x)\right) & =e^{-n \beta} \varphi\left(b \alpha^{-n}(a)\right)=\varphi\left(\alpha^{n}(b) a\right) \\
& =\varphi\left(a \alpha^{n}(b)\right) \\
& =\psi(x y) .
\end{aligned}
$$

Assume next that $\beta$ is infinite, specifically let $\beta=+\infty$. It is then enough to show that for each $x, y \in\left\{a u^{n} \mid a \in A, a u^{n} \in B\right\}$ that the function

$$
z \rightarrow \psi\left(y \varrho_{z}(x)\right)
$$

is bounded in the upper half plane, [2]. But if $x=a u^{n}, y=b u^{m}$, then

$$
\psi\left(y \varrho_{z}(x)\right)=e^{i n z} \psi\left(b \alpha^{m}(a) u^{m+n}\right)
$$

and hence $\psi\left(y \varrho_{z}(x)\right)=0$ unless $m=-n$. When $m=-n$ one has

$$
\begin{aligned}
\psi\left(y \varrho_{z}(x)\right) & =e^{i n z} \varphi\left(b \alpha^{-n}(a)\right) \\
& =e^{i n z} \varphi\left(\alpha^{-n}(a) b\right) .
\end{aligned}
$$

To finish the argument we have to show that $\varphi\left(\alpha^{-n}(a) b\right)=0$ when $n$ is negative. Let $e$ be the projection in $A$ which is the identity for $B$. Then

$$
e x=x e=x,
$$


i.e.

$$
e a u^{n}=a \alpha^{n}(e) u^{n}=a u^{n},
$$

i.e.

$$
e a=a \alpha^{n}(e)=a,
$$

and correspondingly

$$
e b=b \alpha^{-n}(e)=b .
$$

Thus

$$
\alpha^{-n}(a) b=\alpha^{-n}(e) \alpha^{-n}(a) b \alpha^{-n}(e) .
$$

But $\varphi(e)=1$ is finite and $\varphi \alpha=0 \varphi$, and hence

$$
\varphi\left(\alpha^{-n}(a) b\right)=0
$$

when $n$ is negative. The case $\beta=-\infty$ is treated similarly.

Suppose now conversely that $\psi$ is a KMS state at value $\beta$. By $\varrho$-invariance $\psi$ has the form

$$
\psi=\varphi \varepsilon,
$$

where $\varphi$ is a state on $B \cap A$.

Assume first that $\beta$ is finite. Then $\varphi$ is a trace on $B \cap A$. If $x=a u, y=b u^{-1}$ are elements in $B$, i.e.

$$
\begin{aligned}
& e a=a \alpha(e)=a, \\
& e b=b \alpha^{-1}(e)=b,
\end{aligned}
$$

then the KMS-condition,

$$
\psi\left(y \varrho_{i \beta}(x)\right)=\psi(x y),
$$

implies that

$$
e^{-\beta} \varphi\left(b \alpha^{-1}(a)\right)=\varphi(a \alpha(b)) .
$$

Putting $b=e \alpha^{-1}(e)$ in this relation gives

$$
e^{-\beta} \varphi\left(e \alpha^{-1}(e a)\right)=\varphi(a \alpha(e) e),
$$

and using the relations $e a=a \alpha(e)=a$, we obtain

$$
e^{-\beta} \varphi\left(e \alpha^{-1}(a) e\right)=\varphi(e a e) .
$$

In particular this relation is valid for all $a \in(B \cap A) \cap \alpha(B \cap A)$. If $g \in A_{F}$ is the projection corresponding to $x \in G_{F}$ mentioned in the beginning of this section, then $g \leqq e, \alpha^{-1}(g) \leqq e$ and hence $g \in(B \cap A) \cap \alpha(B \cap A)$. It follows that,

$$
e^{-\beta} \varphi\left(e \alpha^{-1}(p) e\right)=\varphi(\text { epe }),
$$

for all projections $p \in A$ such that $p \leqq g$. Since $B \cap A=e A e$ is a hereditary subalgebra of $A$, two projections in $B \cap A$ are equivalent in $B \cap A$ if and only if they are 
equivalent in $A$. It follows from the relation above and the normalization $\varphi(e)=1$ that $\varphi$ extends uniquely to a densely defined lower semicontinuous trace, $\varphi$, on $A$ with

$$
e^{-\beta} \varphi\left(\alpha^{-1}(a)\right)=\varphi(a) .
$$

To see this, observe that it is enough to show that $\varphi$ has a unique extension with these properties when considered as a linear functional on the dimension group of $A$. As all projections $p$ in $A$ corresponding to elements $x^{n}$ in $\mathbb{Z}[x]_{F}^{+}$satisfy the requirements $p \leqq g, \varphi$ is already determined on these. We've shown, in the proof of Theorem 2.4 , that $\varphi$ admits only one scaled extension to all of $G_{F}^{+}$, once it is known on $\mathbb{Z}[x]_{F}^{+}$.

It follows from Theorem 2.4 that

$$
e^{-\beta}\left(1+e^{-\beta}\right)^{-1} \in F,
$$

and that $\varphi$ is the trace defined by $e_{e^{-\beta}}$. Since $\psi=\varphi \varepsilon, \psi$ is unique.

Assume next that $\beta$ is infinite, for example $\beta=+\infty$, and let $a u, b u^{-1} \in B$. Then

$$
\psi\left(a u \varrho_{z}\left(b u^{-1}\right)\right)=e^{-i z} \varphi(a \alpha(b))
$$

is bounded in the upper halfplane, and hence

$$
\varphi(a \alpha(b))=0 .
$$

It follows that

$$
\varphi(e a \alpha(e) b e)=\varphi\left(e a \alpha(e) \alpha\left(e \alpha^{-1}(b) \alpha^{-1}(e)\right)\right)=0
$$

for all $a, b \in A$. The ideal generated by $\alpha(e)$ in $A$ is $I_{+1,-1}$, and hence $\varphi$ vanishes on $e I_{+1,-1} e=e I_{0,-1} e$, which is an ideal in $e A e$. If $0 \notin F$ this ideal is all of $B \cap A$ and $\psi=0$. If $0 \in F$ this ideal has codimension 1 in $B \cap A$, hence $\varphi$ is unique, and $\varphi$ is in fact the trace corresponding to $e_{0}$. Since $\psi=\varphi \varepsilon, \psi$ is unique. The case $\beta=-\infty$ is treated similarly.

3.3. Remark. For finite $\beta$ one has uniqueness of the KMS weight at inverse temperature $\beta$ on the whole crossed product of $A$ by $\alpha$, and this weight is densely defined. For infinite $\beta$ one does have infinitely many ground weights if $0 \in F$, and none of these are densely defined. To substantiate these statements one has to give a precise definition of what is meant by a $\mathrm{KMS}$ weight at value $\beta$, and we treat the cases of finite and infinite $\beta$ separately.

Consider first finite $\beta$. Let $D$ be the crossed product of $A$ by $\alpha$. If $\psi$ is a weight on $D$, we use $D \psi$ to denote its domain and set

$$
D_{2}^{\psi}=\left\{x \in D, \psi\left(x^{*} x\right)<+\infty\right\} .
$$

We say that $\psi$ is a $\varrho$-KMS weight at value $\beta$ if

1. $\psi$ is lower semicontinuous.

2. $\psi$ is $\varrho$-invariant.

3. If $x \in D_{2}^{\psi}$ and the function $t \mapsto \psi\left(x^{*} \varrho_{t}(x)\right)$ has an extension $F$ to the strip

$$
\mathscr{D}_{\beta}=\{z, 0 \leqq \operatorname{sign} \beta \cdot \operatorname{Im} z \leqq \operatorname{sign} \beta \cdot \beta\}
$$


continuous on the boundary, then $x^{*} \in D_{2}^{\psi}$ and

$$
\psi\left(\varrho_{t}(x) x^{*}\right)=F(t+i \beta) .
$$

(This definition is stronger than the definition in [3], see below.)

Assume that $\psi$ is a KMS weight at value $\beta$ on $D$. We first show that

$$
\psi(\varepsilon(x))=\psi(x)
$$

for $x \geqq 0$, where

$$
\varepsilon(x)=\int_{\mathbb{T}} \gamma_{z}(x) d z
$$

is the canonical projection from $D$ into $A$.

First note that we can find a sequence $\left(x_{n}\right)$ of convex combination of translates of $x$ by $\varrho$ converging to $\varepsilon(x)$, and by invariance of $\psi$ we have $\psi\left(x_{n}\right)=\psi(x)$ for all $n$. Since $\psi$ is lower semicontinuous this implies

$$
\psi(\varepsilon(x)) \leqq \psi(x)
$$

for all $x \geqq 0$.

Let $\varphi$ be the restriction of $\psi$ to $A$. It follows immediately from the KMS condition that $\varphi$ is a trace.

If $x \in D$ is a positive element with $\psi(\varepsilon(x))<+\infty$, consider the Fourier expansion

$$
x^{1 / 2} \sim \sum_{n} a_{n} U^{n}
$$

of $x^{1 / 2}$. One has

$$
x=x^{1 / 2} x^{1 / 2 *} \sim \sum_{n, m} a_{n} U^{n} U^{-m} a_{n}^{*}
$$

and hence

$$
\varepsilon(x) \sim \sum_{n} a_{n} a_{n}^{*} .
$$

It follows that $a_{n} a_{n}^{*} \leqq \varepsilon(x)$ for each $n$, hence

$$
\varphi\left(a_{n} a_{n}^{*}\right)=\psi\left(a_{n} a_{n}^{*}\right) \leqq \psi(\varepsilon(x))<+\infty
$$

and since $\varphi$ is a trace

$$
\varphi\left(a_{n}^{*} a_{n}\right)<+\infty
$$

i.e. $a_{n} \in A_{2}^{\varphi} \cap A_{2}^{\varphi *}$ for all $n$.

Let $f_{n}$ be a sequence of functions on $\mathbb{T}=\mathbb{R} / 2 \pi \mathbb{Z}$ with the following properties

1. Each $f_{n}$ is positive.

2. $\frac{1}{2 \pi} \int_{0}^{2 \pi} f_{n}(t) d t=1$.

3. The Fourier transforms $\hat{f}_{n}$ has finite support in $\hat{\mathbb{T}}=\mathbb{Z}$.

4. $\lim _{n \rightarrow \infty} \frac{1}{2 \pi} \int_{0}^{2 \pi} f_{n}(t) g(t) d t=g(0)$ for all continuous functions $g$ on $\mathbb{T}$. 
Define

$$
\begin{aligned}
z_{n} & =\frac{1}{2 \pi} \int_{0}^{2 \pi} f_{n}(t) \gamma_{t}\left(x^{1 / 2}\right) d t \\
& =\sum_{k} \hat{f}_{n}(k) a_{k} U^{k}=\sum_{k} \overline{\hat{f_{n}}(k)} U^{-k} a_{k}^{*},
\end{aligned}
$$

where the last sum is finite. Then $x^{1 / 2}=\lim _{n \rightarrow \infty} z_{n}$ in norm, and hence $x=\lim _{n \rightarrow \infty} z_{n}^{2}$ in norm. But

$$
z_{n}^{2}=z_{n} z_{n}^{*}=\sum_{k, i} \hat{f}_{n}(k) a_{k} U^{k} U^{-i} a_{i}^{*} \overline{\hat{f}_{n}(i)}
$$

and hence

$$
\varepsilon\left(z_{n}^{2}\right)=\sum_{k} \hat{f}_{n}(k) \overline{\hat{f}_{n}(k)} a_{k} a_{k}^{*} \leqq \varepsilon(x) .
$$

[One has $\left|\hat{f}_{n}(k)\right| \leqq 1$ because $\left\|f_{n}\right\|_{1}=1$.] Thus $z_{n}^{2} \in D \psi$ and

$$
\psi\left(z_{n}^{2}\right)=\psi\left(\varepsilon\left(z_{n}^{2}\right)\right) \leqq \psi(\varepsilon(x)) .
$$

It follows from the lower semicontinuity of $\psi$ in the limit $n \rightarrow \infty$ that

$$
\psi(x) \leqq \psi(\varepsilon(x)) .
$$

We have proved that $\psi(\varepsilon(x)) \leqq \psi(x)$ for all $x \geqq 0$, and $\psi(x) \leqq \psi(\varepsilon(x))$ whenever $x \geqq 0$ and $\psi(\varepsilon(x))<+\infty$. It follows that

$$
\psi(\varepsilon(x))=\psi(x)
$$

for all $x \geqq 0$. This shows that $\psi$ is uniquely determined by $\varphi$.

If $a \in A_{2}^{\varphi}$, define $x=U a$. Then

$$
\psi\left(x^{*} x\right)=\psi\left(a^{*} U^{*} U a\right)=\psi\left(a^{*} a\right)=\varphi\left(a^{*} a\right)<+\infty
$$

and hence $x \in D_{2}^{\psi}$. Since $\varrho_{t}(x)=e^{i t} x$ the function

$$
F(z)=e^{i z} \psi\left(x^{*} x\right)
$$

is an entire analytic extension of

$$
t \rightarrow \psi\left(x^{*} \varrho_{t}(x)\right)
$$

and it follows from the KMS condition that $x^{*} \in D_{2}^{\psi}$ and

$$
e^{-\beta} \psi\left(x^{*} x\right)=\psi\left(x x^{*}\right)
$$

i.e.

$$
e^{-\beta} \psi\left(a^{*} U^{*} U a\right)=\psi\left(U a a^{*} U^{*}\right)
$$

or

$$
\left.e^{-\beta} \varphi\left(a^{*} a\right)=\varphi\left(\alpha\left(a a^{*}\right)\right)=\varphi\left(a^{*} a\right)\right)
$$

for all $a \in A_{2}^{\varphi}$. It follows that $A_{2}^{\varphi}$ is $\alpha$-invariant and

$$
\varphi \alpha=e^{-\beta} \varphi \text {. }
$$


It follows from 2.4 that $\varphi$ is unique, and hence $\psi$ is unique, up to normalization.

Note that if one replaces condition 3 in the definition of a KMS weight $\psi$ by the more conventional condition given by

3'. If $x, y \in D_{2}^{\psi} \cap D_{2}^{\psi *}$, then there exists a function $F$, analytic in $\mathscr{D}_{\beta}$ and continuous on its boundary, such that

$$
\begin{aligned}
F(t) & =\psi\left(y \varrho_{t}(x)\right) \\
F(t+i \beta) & =\psi\left(\varrho_{t}(x) y\right),
\end{aligned}
$$

then the KMS-weight at value $\beta$ is no longer unique. If for example $\psi$ is the KMS weight considered above and $C$ is a $\varrho$-invariant hereditary $C^{*}$-subalgebra of $D$, one can define a new weight $\psi^{\prime}$ by

$$
\psi^{\prime}(x)=\psi(x),
$$

where $x \geqq 0$ and $x \in D^{\psi} \cap C$, and

$$
\psi^{\prime}(x)=+\infty
$$

for all other $x \geqq 0$. One verifies easily that $\psi^{\prime}$ is a KMS weight at value $\beta$ in the sense of $3^{\prime}$. This lack of uniqueness is generic. That is, if one adopts $3^{\prime}$ as the definition for a KMS weight, the weight must agree with a densely finite one, where defined. We will not prove this here.

The non-uniqueness of KMS weights on $D$ for infinite inverse temperatures can be seen as follows: If $0 \in F$ define additive linear functionals $e_{0}^{n}: G_{F} \rightarrow \mathbb{R} \cup\{ \pm \infty\}$ by $e_{0}^{n} a=\lim _{t \rightarrow 0} t^{n} a(t), a \in G_{F}$. These give rise to weights on $A_{F}$, also denoted by $e_{0}^{n}$, such that $e_{0}^{n} \alpha(a)=0 \cdot e_{0}^{n}(a)$ provided $e_{0}^{n}(a) \neq \pm \infty$. The kernel of the associated representation is $I_{\infty, n-1}$ and thus the weights for different $n$ are distinct. They all are KMS weights at $+\infty$, these weights are not finite on a norm dense set of the crossed product. Note that the restriction of $e_{0}^{n}$ to the ideal $I_{\infty, n}$ is densely defined and lower semicontinuous, while $e_{0}^{n}(a)=+\infty$ whenever $a$ is a positive element in $A_{F} \backslash I_{\infty, n}$. Thus the restriction of $e_{0}^{n}$ to $A_{F}$ is lower semicontinuous, and $e_{0}^{n} s$ is lower semicontinuous on $D$.

The uniqueness of KMS states on the system $(B, \varrho)$ for infinite inverse temperatures is due to the fact that if 0 or 1 is in $F$ then $B \cap A_{F}$ has maximal ideals of codimension one. This system can be modified so that for $\beta= \pm \infty$ one has nonuniqueness of $\beta$-KMS states, as follows. Assume that 0 or 1 is in $F$, and consider the tensor product of this system with the trivial one-parameter automorphism group on a simple unital $C^{*}$-algebra $R$ which has a unique tracial state. Then if $0 \in F($ resp. $1 \in F)$, the set of $\beta$-KMS states at $\beta=+\infty$ (resp. $\beta=-\infty$ ) of the tensor product system is naturally isometric to the state space of $R$.

3.4. Up to this point we have illustrated how to obtain $C^{*}$-algebra with $\mathrm{KMS}$ states at unique values. In fact non-uniqueness can also be specified. The first result is arrived at by modifying the techniques above.

Theorem 3.4.1. Let $K_{1} \supseteqq K_{2} \supseteqq \ldots \supseteqq K_{n}$ be a decreasing finite sequence of closed subsets of $\mathbb{R} \cup\{ \pm \infty\}$ such that $\pm \infty \notin K_{2}$. Then there exists a unital, separable, simple, nuclear $C^{*}$-algebra $\mathscr{B}$ and a one-parameter, *-automorphism group, $t \rightarrow \varrho_{t}$, of 
period $2 \pi$, acting on $\mathscr{B}$. For this automorphism group there is a $\varrho$-KMS state on $\mathscr{B}$ at value $\beta$ if and only if $\beta \in K_{1}$. Moreover if $\beta \in K_{k} \backslash K_{k+1}\left(K_{n+1} \equiv \phi\right)$ then the set of $(\varrho, \beta)-\mathrm{KMS}$ states is isomorphic to the finite simplex with $k$ extreme points, $k=1, \ldots, n$.

Proof. As in the proof of Theorem 3.2 consider the closed subsets of $[0,1]$, $F_{k}=\left\{e^{-\beta}\left(1+e^{\beta}\right)^{-1}, \beta \in K_{k}\right\}$. With $F=\left(F_{1}, F_{2}, \ldots, F_{n}\right)$ define a group $G_{F}$ by

$$
G_{F}=\bigoplus_{k=1}^{n} \mathbb{Z}\left[x, x^{-1},(1-x)^{-1}\right]_{F_{k}}
$$

with the order defined by $p=\bigoplus_{k=1}^{n} p_{k}>0$ if and only if $p_{k}>0$ as an element in $G_{F_{k}}$. The rest of the proof is as in Theorem 3.2.

One can, in fact, deal not only with finite-dimensional simplexes but metrizable ones. We write $K_{\beta}$ for the set of KMS states at inverse temperature $\beta$.

Theorem 3.4.2. Let $\beta_{1}, \beta_{2}, \ldots, \beta_{n} \in \mathbb{R}$ be $n$-distinct numbers and $K_{1}, K_{2}, \ldots K_{n}$ be $n$, compact, metrizable simplexes. There exists a $C^{*}$-dynamical system $(\mathscr{B}, \gamma, \mathbb{R})$ where $\mathscr{B}$ is unital and simple and $\gamma$ is of period $2 \pi$, such that $\mathscr{B}$ has $\beta$-KMS states if and only if $\beta \in\left\{\beta_{1}, \ldots, \beta_{n}\right\}$ and $K_{\beta_{k}} \cong K_{k}$ for $k=1, \ldots, n$.

Proof. Suppose first that $n=1$. Note that the additive group $A\left(K_{1}\right)$ of all real, affine, continuous functions on $K_{1}$ is a Riesz group in the usual ordering, since $K_{1}$ is a simplex. To get a simple $C^{*}$-algebra, however, we need a stronger ordering. Define $a>0$ if and only if $a(\omega)>0$ for all $\omega \in K_{1}$. Because of compactness of $K_{1}$, $A\left(K_{1}\right)$ is still a Riesz group. Take as a dimension group $G$, any countable norm dense subgroup of $A\left(K_{1}\right)$ which contains the constant function 1, which is a Riesz group in the inherited ordering, and further is invariant under multiplication by the scalars $e^{ \pm \beta_{1}}$. Let $A$ be the $A F$-algebra corresponding to $G$ and $\alpha$ the automorphism of $A$ which arises from the order preserving automorphism of $G$ given by multiplication by $e^{-\beta_{1}}$. Let $\mathscr{B}$ denote the crossed product of $A$ by $\alpha$ cut down by a projection corresponding to 1 in $G$ (except when $\beta_{1}=0$ where we take $\mathscr{B}=A$ cut down by [1] and $\gamma=1$ ) and take $\gamma$ to be the restriction of the dual automorphism, on $C^{*}(A, \alpha)$, to $\mathscr{B}$. The rest of the proof proceeds as before, for $n=1$.

To treat the case $n \geqq 2$ we follow the idea in Theorem 3.4.1. Construct groups $G_{1}, \ldots, G_{n}$ by the recipe in the paragraph above and then form $G=\bigoplus_{k=1}^{n} G_{n}$ with the order $a=\bigoplus_{k=1}^{n} a_{k}>0$ if and only if $a_{k}>0$ in $G_{k}$ for all $k$. The automorphism $\alpha$ is given by $\alpha=\bigoplus_{k=1}^{n} \alpha_{k}$. One then constructs $A$ as Theorem 3.4.1.

3.5. Remark. Many of the preceding arguments are valid in a more general context. For instance, one sees that the dual automorphism group of an automorphism $\alpha$ of any $C^{*}$-algebra $A$ has a ground state (resp. ceiling state) on the crossed product if and only if there is a non-trivial closed two-sided ideal of $A$ taken into a subset of itself by $\alpha$ (resp. by $\alpha^{-1}$ ), as examining the proof of Theorem 3.2 will show. In particular, one concludes that the fixed point subalgebra of any periodic automorphism group with a ground state is not simple. 
(Use the duality of [18] and the result of Rosenberg in [15] that the fixed point algebra of a compact group is isomorphic to a hereditary sub- $C^{*}$-algebra of the crossed product, generating an essential closed two-sided ideal.) This is not true in general, i.e. without periodicity; as can be seen by considering quasi-free automorphisms of the CAR algebra. For finite $\beta$ simplicity of the fixed point algebra is allowed, of course. Both results could also be proved more directly. In fact we now give a direct proof of the second statement above.

One sees that the algebra $A^{\prime \prime}=" \oplus A(n)$ where the $A(n)$ are eigenspaces corresponding to $e^{\text {int }}$. Then $A(0)$ is a sub- $C^{*}$-algebra of $A$, the fixed point algebra of $\alpha_{t}$. If $A(0)$ is simple and $A(-n) \neq 0$ for some $n$, then the ideal $A(-n)^{*} A(-n)$ is dense in $A(0)$. Then $I$ is approximated by elements $\sum y_{i}^{*} x_{i}, y_{i}, x_{i} \in A(-n)$. But if $x \in A(-n)$, $\omega\left(x^{*} \alpha_{t}(x)\right)$ does not extend to a bounded analytic function in the upper half plane unless $\omega\left(x^{*} x\right)=0$. Thus $\omega\left(\sum y_{i}^{*} x_{i}\right)=0$. But then $\omega(I)=0$, a contradiction. As $n$ was arbitrary, this makes the automorphism group trivial.

3.6. Remark. So far only nonempty closed sets of inverse temperatures have been considered. To get a $C^{*}$-dynamical system with no KMS states at all, take a system in which the only inverse temperature is 0 , for example that of 3.1 with $F=\left\{\frac{1}{2}\right\}$ (alternatively, one can take the example of Lance and Niknam in [10], which they showed has no ground state, and which is easily seen as in 3.2 , to have a $\beta$-KMS state only when $\beta=0$ ), and take the tensor product of this system with the trivial automorphism group on a simple unital $C^{*}$-algebra with no trace (for example, a factor of type III or the algebra $\mathrm{O}_{2}$ of [4]).

\section{Remarks on the Powers-Sakai Conjecture}

Recall, [12], that the conjecture states that every one-parameter *-automorphism group of a UHF $C^{*}$-algebra is approximately inner.

4.1. If $F=[0,1]$, then $B$ is the Fermion $C^{*}$-algebra (see [1, 14, Appendix], and $\gamma$ is the gauge group, an approximately inner group.

4.2. If $0 \notin F$ then, by $3.2, B$ does not have a ground state for $\varrho$ and so by 2.3 of [12], $\varrho$ is not approximately inner. Since 2.3 of [12] is also valid for ceiling states, $1 \notin F$ also implies that $\varrho$ is not approximately inner. (This idea yields a short proof of the fact proved in [11] that the dual automorphism group in the $C^{*}$-algebra $O_{\infty}$ of Cuntz is not approximately inner.)

If $\frac{1}{2} \in F$ but $F \neq[0,1]$ then, by $3.2, B$ has a tracial state but does not have KMS states for $\varrho$ at arbitrary nonzero inverse temperatures. Therefore by Theorem 3.2 of [12], $\varrho$ is not approximately inner.

4.3. The case $\frac{1}{2} \in F$ shows that one can have a simple, finite amenable $C^{*}$-algebra where the Powers-Sakai conjecture does not hold. Another example of this is the irrational rotation algebra where translation on the circle is lifted to this crossed product. If the automorphism group is approximately inner then a ground state exists [12, Theorem 2.3] but this must then be a ground state for the automorphism restricted to $C(T)$, an impossibility [17].

The argument used to deal with the irrational rotation algebra leads to the following 
Proposition 4.3.1. Let $(\mathfrak{A}, \tau, \mathbb{R})$ be a $C^{*}$-dynamical system, $\omega$ a KMS state at value $\beta \in \mathbb{R} \backslash\{0\} \cup\{ \pm \infty\}$. Suppose that $\pi_{\omega}$ is faithful. If $\mathscr{B} \subseteq \mathfrak{U}$ is an abelian * subalgebra left globally invariant by $\tau$, i.e. $\tau_{t}(\mathscr{B}) \cong \mathscr{B}$, then $\tau_{t}(B)=B$ for all $B \in \mathscr{B}$.

Proof. If $\beta$ is finite then the KMS condition applied only to elements in $\mathscr{B}$ yields the result immediately. If $\beta$ is $\pm \infty$ then recall that Borcher's theorem requires the corresponding dynamics to be inner in the abelian von Neumann algebra $\pi_{\omega}(\mathscr{B})$.

4.4. The "first step" in trying to show that an automorphism group $t \rightarrow \alpha_{t}$ of a $C^{*}$-algebra is approximately inner is to show that unitary eigenoperators do not exist i.e. there is no unitary operator $u \in \mathfrak{A}$ such that, $(+) \alpha_{t}(u)=e^{i \lambda t} u$, unless $\lambda=0$. That this cannot occur for UHF $C^{*}$-algebras was pointed out to us by $\mathrm{A}$. Kishimoto. In fact he observed that a theorem of Pusz and Woronowicz [13, Theorem 2.1] gives the result immediately. If we write $\delta$ for the generator of $\alpha_{t}$ and $\tau$ for the trace on $\mathfrak{A}$, they show that the function $u \rightarrow \tau\left(u^{*} \delta(u)\right)$ vanishes on the connected component of the unitary group. Since for UHF $C^{*}$-algebras the unitary group is connected $(+)$ cannot occur unless $\lambda=0$. This idea, however, shows much more.

Proposition 4.4.1. If $\mathfrak{U}$ is a unital AF algebra then it is not the crossed product of any $C^{*}$-algebra by the integers.

Proof. The unitary group of an $A F$ algebra is connected. The dual automorphism group and the construction of the crossed product provides a unitary and an automorphism group satisfying $(+)$ with $\lambda=1$, since if the crossed product is a unital $A F$ there is a finite trace which then may be averaged to yield an invariant trace for the dual action.

This proposition yields the "known" fact that the irrational rotation algebra is not $A F$.

Acknowledgement. We would like to thank A. Kishimoto for several fruitful discussions. The first and third author (O.B. and R.H.H.) are grateful to Professors D. Kastler and M. Mebkhout for arranging visits to Luminy, where part of this work was done.

\section{References}

1. Bratteli, O. : Crossed products of UHF algebras by product type actions. Duke Math. J., 46 (1), 123 (1979)

2. Bratteli, O., Robinson, D.: Operator algebras and quantum statistical mechanics, Vol. 2. Berlin, Heidelberg, New York: Springer-Verlag (to appear)

3. Combes, F.: Poids et espérances conditionelles dans les algèbras de von Neumann. Bull. Soc. Math. France 99, 73-112 (1971)

4. Cuntz, J.: Simple $C^{*}$-algebras generated by isometries. Commun. Math. Phys. 57, 173-185 (1977)

5. Effros, E., Handelman, D., Shen, C.-L.: Dimension groups and their, affine representations. American J. Math. 102, 385-408 (1980)

6. Elliott, G.A.: On the classification of inductive limits of sequences of semisimple finite-dimensional algebras. J. Algebra 38, 29-44 (1976)

7. Elliott, G.A.: Some simple $C^{*}$-algebras constructed as crossed products with discrete outer automorphism groups. Publ. Res. Inst. Math. Sci. (to appear)

8. Fuchs, L.: Riesz groups. Ann. Scuola Norm. Pisa III 19, 1-34 (1965)

9. Jørgensen, P.: Trace states and KMS states for approximately inner dynamical one-parameter groups of *-automorphisms. Commun. Math. Phys. 53, 135-142 (1977) 
10. Lance, E.C., Niknam, A.: Unbounded derivations of group $C^{*}$-algebras. Proc. Am. Math. Soc. 61, 310-314 (1976)

11. Olesen, D., Pedersen, G.K.: Some $C^{*}$-dynamical systems with a single KMS state. Math. Scand. 42, 111-118 (1978)

12. Powers, R., Sakai, S.: Existence of ground state and KMS states for approximately inner dynamics. Commun. Math. Phys. 39, 273-288 (1975)

13. Pusz, W., Woronowicz, S.L.: Passive states and KMS states for general quantum systems. Commun. Math. Phys. 58, 273-290 (1978)

14. Renault, J.: A Groupoid approach to $C^{*}$-algebras. Thesis, Berkeley 1978

15. Rosenberg, J. : Appendix to O. Bratteli's paper on crossed products of UHF algebras. Duke Math. J. 46, 25-26 (1979)

16. Shen, C.-L.: On the classification of the ordered groups associated with the approximately finite dimensional $C^{*}$-algebras. Duke Math. J. 46, 613-633 (1979)

17. Sakai, S.: The theory of unbounded derivations. In: Mimeographed Lecture Notes, The University, Newcastle-upon-Tyne, and The University of Copenhagen 1978

18. Takai, H.: On a duality for crossed products of $C^{*}$-algebras. J. Functional Analysis 19, 25-39 (1975)

19. Takesaki, M.: Lectures on operator algebras. Mimeographed Notes, UCLA, 1971

Communicated by $\mathrm{H}$. Araki

Received July 16, 1979; in revised form February 5, 1980 
